\title{
ANÁLISIS \\ El proyecto transregional de la administración Trump y la competencia por la integración económica en el Pacífico
}

\author{
The Trump's Administration Trans-Regional Project and the \\ Competition for Economic Integration in the Pacific
}

DOI: $10.32870 /$ mycp.v10i30.748

\section{Resumen}

En este texto se propone una lectura particular del proyecto transregional Asia del Pacífico-América del Norte-Europa (diseñado y puesto en práctica parcialmente por los asesores comerciales de Donald Trump) y de las condiciones en que enfrenta la competencia política del Comprehensive and Progressive Trans Pacific Partnership Agreement (CPTPP, encabezado por el Gobierno japonés) y del Regional Comprehensive Economic Partnership (RCEP, liderado por el Gobierno chino). En ese marco, el artículo destaca los alcances de cada una de esas iniciativas en materia de regulación comercial, y, recurriendo al ejemplo de Japón, muestra cómo cada gobierno está dispuesto a hacer concesiones comerciales a sus contrapartes, en función de cada socio y de cada acuerdo comercial. En el trabajo se propone que los asesores de la administración Trump marcaron un giro al abandonar la formación de capital, para adoptar el consumo de las familias y del Gobierno, como la principal ventaja comparativa de la economía estadounidense; en ese nuevo contexto, el acceso a los mercados estadounidense y/o norteamericano, bajo condiciones privilegiadas, tiene como costo aceptar y cumplir las regulaciones de los acuerdos bilaterales con Estados Unidos y/o del United StatesCanada-Mexico Agreement (USMCA). El autor concluye que las contrapartes asiáticas y norteamericana del Gobierno estadounidense necesitan revisar las relaciones comerciales entre ellos para cerrar el círculo de los acuerdos bilaterales y beneficiarse de la iniciativa estadounidense.

Palabras clave: competencia comercial, acuerdos comerciales, liberación comercial, ventajas competitivas, integración económica.

\author{
Juan José Ramírez Bonilla ${ }^{1}$
}

\begin{abstract}
The text proposes a particular reading of the Asia Pacific-North America-Europe integration project (designed and partially implemented by Donald Trump's trade advisers) and the conditions under which it faces the political competition of the Comprehensive and Progressive Trans-Pacific Partnership (CPTPP, headed by the Japanese government) and the Regional Economic Comprehensive Partnership (RCEP, led by the Chinese government). In this context, the article highlights the scope of each of these trade regulation initiatives, and, using Japan's example shows how each government is willing to make trade concessions to its counterparties, depending on each partner and each trade agreement. The work proposes that advisers to the Trump administration set a turn by abandoning capital formation, to embrace the consumption of families and government as the main comparative advantage of the US economy. In this new situation, access to the US and/or to the North American markets, underprivileged conditions, costs to accept and comply with the regulations of bilateral agreements and/or the United States-CanadaMexico Agreement (USMCA). The author concludes that Asian and North American partners of the US government should review trade relations between them to close the circle of bilateral agreements and benefit from the Us initiative.
\end{abstract}

Keywords: commercial competition, trade agreements, commercial liberalization, competitive advantages, economic integration.

Artículo recibido el 09 de febrero de 2021 y dictaminado el 03 de mayo de 2021.

1. El Colegio de México, A. C. (Colmex). Carretera Picacho Ajusco 20, Col. Ampliación Fuentes del Pedregal, C. P. 14110, Tlalpan, Ciudad de México, México. ORcid: https://orcid.org/0000-00016109-2269. Correo electrónico: jrami@colmex.mx 
La firma del Regional Comprehensive Economic Partnership (RCEP), ${ }^{2}$ propuesto por los gobiernos participantes en la Association of South East Asian Nations (ASEAN) e impulsado por el Gobierno de la República Popular China (en adelante, China), ha despertado un gran interés entre los comunicadores, los funcionarios y los académicos mexicanos.

Las justificaciones comunes de ese interés son la importancia cuantitativa del RCEP en el contexto de la economía mundial y el papel del Gobierno chino en las negociaciones del acuerdo; en efecto, el RCEP es considerado "la mayor asociación comercial del mundo", en la medida en que "representa una gran victoria para China, el principal promotor del proyecto [...], y abarcará a $2 \mathrm{mil}$ 100 millones de consumidores y 30 por ciento del PIB mundial" (Ruiz, 2020).

Ahora bien, si tenemos en cuenta el tamaño de la población del conjunto de países cuyos gobiernos firmaron el RCEP, sin duda el acuerdo integrará, en un lapso de 35 años, el mercado más grande del mundo; en 2018 los países del RCEP representaban $30.11 \%$ de la población mundial (United Nations, 2019) y la proporción habría aumentado hasta $47.45 \%$ si el Gobierno indio se hubiera sumado a la iniciativa.

Desde la perspectiva económica, no obstante, la importancia del RCEP necesita ser matizada; un primer matiz deriva de la forma de considerar las informaciones sobre el producto interno bruto (PIB) y sobre el comercio total (ст, o la suma de los valores de las exportaciones y de las importaciones) correspondientes a 2018, para los grandes proyectos regionales en curso, tanto en el Pacífico como en Europa.

Así, en el Pacífico el RCEP supera de manera diferenciada acuerdos como el United States-Canada-Mexico Agreement (USMCA) y el Comprehensive and Progressive Trans Pacific Partnership Agreement (CPTPP). Desde el punto de vista de la producción, la supremacía del RCEP es prácticamente marginal sobre el USMCA y muy amplia sobre el CPTPP; en efecto, las participaciones de cada uno de esos acuerdos comerciales en el valor del PIB total mundial fueron respectivamente 29.91, 27.61 y $12.79 \%$. En contraste, la superioridad comercial del RCEP es incontestable, pues en 2018 aportaba el $29.37 \%$ del CT mundial, mientras las participaciones del USMCA y del CPTPP eran 16.13 y $14.48 \%$, respectivamente.

2. Los 15 jefes de Estado y de Gobierno participantes en el RCEP firmaron el acuerdo el 15 de noviembre de 2020, durante la reunión virtual del 4th RCEP Summit (ASEAN, 2020). 
Ahora bien, en relación con Europa la situación era muy diferente; la Unión Europea (UE) aportaba el 30.60\% del PIB total mundial y el $29.37 \%$ del CT mundial. En 2018 existía una situación de igualdad productiva y comercial entre el RCEP y la UE; cierto, las expresiones políticas de la competencia comercial entre chinos y europeos son mucho menos crispadas que entre chinos y estadounidenses; además, las relaciones sino-europeas suelen quedar fuera del horizonte de los observadores mexicanos.

Hasta aquí nos hemos apegado al canon de análisis prevaleciente en México, y según el cual los procesos extrarregionales eran vistos a través del North American Free Trade Agreement (NAFTA), y ahora lo son en la perspectiva del USMCA. Sin embargo, para tener una idea completa de las condiciones de la competencia entre los grandes proyectos regionales necesitamos ubicarnos en la perspectiva de las potencias involucradas; en ese sentido, nuestro trabajo tiene como hilo conductor la iniciativa de integración transregional de la administración Trump; este proyecto incluía originalmente la negociación de acuerdos bilaterales con México y Canadá (sintetizados en el USMCA), con Japón, Corea y Filipinas (en Asia del Pacífico) y con la Unión Europea y el Reino Unido (en Europa); cierto, la victoria electoral de Joe Biden proyecta una sombra de incertidumbre sobre la manera en que habrá de administrar los acuerdos comerciales bilaterales negociados con los cuatro primeros gobiernos mencionados, así como sobre la actitud ante las negociaciones comerciales con Filipinas, la Unión Europea y el Reino Unido.

En todo caso, para nuestros fines interesa particularmente la competencia entre las tres iniciativas macrorregionales de integración económica en curso, en la región del Pacífico: el proyecto transregional de la administración Trump (en el estado de operación que se encuentra hasta este momento), el RCEP y el CPTPP. Para dar cuenta de las condiciones en que los promotores de esa iniciativa enfrentan la competencia de sus rivales, hemos dividido nuestro texto en dos partes: en la primera, analizamos las características generales de las tres propuestas macrorregionales, destacando sus orígenes y objetivos iniciales, así como las ventajas económicas y políticas que ofrecen a sus participantes. En la segunda parte estudiamos los contenidos del CPTPP, del RCEP y, a título de ilustración del alcance del proyecto de la administración Trump, del USMCA y/o del Japan-United States Trade Agreement (JUSTA). En el ámbito general, destacamos las áreas cubiertas por los textos de cada acuerdo, para insistir en que el RCEP es el menos sofisticado de los tres y que el USMCA incorpora un mayor número de temas nuevos, lo cual lo torna en el acuerdo comercial 
más complejo. En el tema de la liberalización comercial, contrastamos los resultados de las negociaciones y mostramos las diferencias en materia de política económica internacional, en función no solo de cada tipo de acuerdo, sino también de cada contraparte participante en un acuerdo específico; así, mediante el ejemplo del Gobierno de Japón, mostramos que en el JUSTA las autoridades japonesas asumen virtualmente una actitud irrestricta de libre comercio; mientras que en el CPTPP todavía mantienen barreras arancelarias y no arancelarias, principalmente en el sector de bienes agropecuarios; esos mecanismos proteccionistas tienen una mayor presencia en el RCEP y su carácter restrictivo va de más a menos con China, con Corea del Sur y con los miembros de la ASEAN. Finalmente, destacamos las principales ventajas comparativas del CPTPP, del RCEP y de las economías participantes en el proyecto transregional estadounidense, para poner en relieve el contraste entre la gran ventaja productiva del RCEP (basada en una formación bruta de capital masiva y en una participación abrumadora en el comercio total mundial) y la ventaja del proyecto transregional de la administración Trump (fundada en el consumo de los hogares y del gobierno, tanto en Estados Unidos como en América del Norte).

\section{Aspectos generales de las tres iniciativas macrorregionales}

Las dos primeras décadas del siglo Xxi han estado marcadas, primero, por la formulación de proyectos de carácter regional en un sentido amplio; la malograda Americas' Free Trade Area (AFTA) provocó reacciones en cadena que llevaron a los gobiernos chino, japonés, coreano, indio, australiano y neozelandés a firmar sendos acuerdos con los 10 participantes en la ASEAN; de estas reacciones y de la experiencia de la segunda mitad de los noventa expresada en la propuesta de la ASEAN sobre la East Asian Community (EAC), resultaron las iniciativas del Comprehensive Economic Partnership for East Asia (CEPEA) y finalmente del RCEP. Mientras tanto, los gobiernos participantes en el Asia-Pacific Economic Cooperation (APEC) transitaron progresivamente del "regionalismo abierto" al proyecto de una Asia-Pacific Free Trade Area (APFTA) (APEC, 2008) que debería incluir las 21 economías de los países participantes en el foro. Finalmente, la decisión de la administración Bush de incorporarse al Trans Pacific Strategic Economic Partnership Agreement (TPSEP) creó las condiciones para las negociaciones del Trans-Pacific Partnership (TPP); la decisión de la administración Trump (en 2016) de retirarse 
de este último acuerdo permitió su renegociación y su transformación en el CPTPP, además desbrozó el camino para la puesta en práctica del proyecto transregional estadounidense.

\subsection{El proyecto transregional de la administración Trump}

Durante los primeros 15 años del siglo xxi, China logró convertirse en el primero o en el segundo socio comercial de los países del Pacífico asiático, desplazando a Japón a un segundo plano y relegando todavía más a Estados Unidos. El ascenso económico de China descansó en gran medida sobre la integración de facto de un complejo productivo regional, integrado por las economías de los países del Pacífico asiático; dicho complejo cristalizó en una organización radial, centrada en la economía china. Para los consejeros comerciales de la administración Trump ese proceso imponía la necesidad de establecer condiciones internacionales análogas, para permitir a la economía estadounidense competir con el gigante asiático en situación de igualdad; el satisfactor de esa necesidad fue el proyecto transregional de integración económica, también organizado radialmente y centrado sobre la economía estadounidense (Ramírez Bonilla, 2020a).

Ahora bien, por regla general los analistas malinterpretaron los propósitos políticos de Donald Trump; cierto, el eslogan America First proponía una nueva forma de proteccionismo; pero la práctica política de la administración Trump demostró que su política económica internacional distaba de ser aislacionista y que pugnaba por redefinir la estructura del orden económico internacional. Para lograr esa redefinición, sus asesores comerciales delinearon un proyecto de integración transregional, organizado radialmente y centrado sobre la economía estadounidense; el proyecto fue definido en tres etapas:

Primero, como candidato republicano a la Presidencia, Donald Trump presentó

siete medidas de la futura política económica internacional: 1 . Retirar a Estados Unidos del TPP; 2. Designar negociadores comerciales severos; 3. Identificar violaciones a los acuerdos comerciales y utilizar las herramientas del derecho estadounidense para poner término a los abusos; 4. Renegociar el Tratado de Libre Comercio de América del Norte (TLCAN) $y$, en caso de una negativa de sus contrapartes, retirarse del acuerdo; las tres medidas restantes concernían directamente a China (Trump, 2016). Segundo, durante el APEC CEO Summit Meeting 2017, realizado en Da Nang, Vietnam, Donald Trump definió oficialmente su política económica in- 
ternacional: "estableceré acuerdos comerciales bilaterales con cualquier nación de [la región] Indo-Pacífico que quiera ser nuestro socio y que cumpla con los principios del comercio justo y recíproco [...]" (Trump, 2017). Tercero, durante 2017 y 2018 renegoció los acuerdos con la República de Corea (firmado, por tercera ocasión, el 24 de septiembre de 2018), con Canadá y con México (firmados el 20 de noviembre de 2018). Entre las fechas de formalización de esos tres acuerdos, el representante comercial de Estados Unidos anunció al Congreso la voluntad de la administración Trump de emprender negociaciones comerciales con Japón, la Unión Europea, Reino Unido y Filipinas (Reuters, 2018). Finalmente, el 7 de octubre de 2019 los representantes comerciales de Japón y de Estados Unidos firmaron el JUSTA (Office of the Us Trade Representative, 2019).

El proyecto de integración transregional de la administración Trump proponía acuerdos comerciales bilaterales con siete entidades políticas; cuatro fueron firmados y han sido heredados por la administración Biden; debido a esa circunstancia, a lo largo de nuestro texto insistimos en la "iniciativa" o en el "proyecto" de integración transregional, para distinguirlo de los acuerdos comerciales que lo integran o de los que están en vigor y con los cuales compite en la región del Pacífico.

En términos formales, todo apuntaba hacia una reacción estadounidense ante la proyección regional y global de la economía china; sin embargo, en términos de contenido la iniciativa de la administración Trump fue innovadora en, cuando menos, cuatro sentidos: en primer término, dejaba de lado las ventajas competitivas tradicionales (productividad, bajos costos y alta calidad de los bienes producidos en Estados Unidos), para privilegiar el acceso al mercado estadounidense, el más importante desde la perspectiva del consumo final; es decir, dejaba de lado las ventajas competitivas basadas en la producción, para utilizar el consumo efectivo de la sociedad estadounidense como factor de atracción para las empresas de los socios en acuerdos comerciales bilaterales; más adelante veremos con más detalle este factor de atracción.

En segundo lugar, los negociadores estadounidenses abandonaron la teórica reciprocidad estructural de las concesiones comerciales que se otorgan dos o más participantes en los llamados acuerdos de libre comercio (regulados por las estipulaciones del texto del General Agreement on Tariffs and Trade —GATT—, en su versión de 1947), obligando a sus contrapartes a satisfacer los intereses comerciales estadounidenses como precio para pagar el acceso 
al mercado de Estados Unidos. En otro lugar hemos denominado "proteccionismo consensuado" al proteccionismo característico de los "acuerdos de libre comercio", y "proteccionismo coercitivo" al proteccionismo practicado por la administración Trump (Ramírez Bonilla, 2020b).

En tercera instancia, fueron definidas dos formas diferenciadas de acceder al mercado estadounidense: la primera es directa: las empresas ubicadas en Corea, Japón, México y Canadá exportan directamente a Estados Unidos; los acuerdos bilaterales definen tanto las condiciones particulares del acceso de los bienes y servicios al mercado estadounidense, como las amplias concesiones realizadas por cada uno de esos gobiernos a su contraparte estadounidense. La segunda manera de acceder al mercado estadounidense es indirecta: las empresas coreanas y japonesas (o de cualquier otro origen nacional) producen en México y en Canadá para exportar al mercado estadounidense; los costos de acceso quedan sujetos a las regulaciones del USMCA.

Finalmente, fue privilegiada la relación con países considerados avanzados, desarrollados o industrializados, de acuerdo con la jerga utilizada; la única excepción es México, debido a su función como base productiva integrada estrechamente a la economía estadounidense.

\subsection{El CPTPP}

Mucha tinta ha corrido para explicar la decisión de la administración Trump de retirarse del Trans Pacific Partnership Agreement (TPP o TPP-12), firmado por la administración Obama y por 11 gobiernos de ambas riberas del Pacífico, el 4 de febrero de 2016. Por nuestra parte, deseamos destacar los siguientes aspectos del TPP:

Primero, desde la campaña electoral de 2016, Donald Trump anunció retirarse de todos los acuerdos comerciales multilaterales, para reemplazarlos por acuerdos bilaterales con los principales socios comerciales; poco después de entrar en funciones, en enero de 2017, puso en práctica su intención de retirar a Estados Unidos del TPP, quedando los 11 gobiernos restantes sin saber qué hacer con el resultado de la negociación; después de algunas semanas de incertidumbre, el Gobierno japonés asumió la dirección de la renegociación del TPP entre los 11 gobiernos restantes, logrando su aprobación después de suspender algunos de los temas considerados prioritarios para el Gobierno estadounidense, pero problemáticos para algunos de los gobiernos 
negociadores. ${ }^{3}$ Para múltiples observadores estas disposiciones afectaban principalmente a los países en desarrollo participantes en el acuerdo, pero también apuntaban directamente contra una economía china insuficientemente madura para ajustarse a esos estándares en caso de que sus autoridades deseasen sumarse al TPP.

Ahora bien, desde febrero de 2013 los negociadores estadounidenses fueron muy claros al señalar que, en el caso de Japón, "no vamos a empezar el proceso [de negociación] diciendo: 'Oh, claro, podrán mantener protegidos algunos sectores o áreas'”, indicando con ello que debían negociar todos los sectores, incluidos los más delicados como el arroz (Palmer, 2013). En todo caso, el Gobierno japonés debió hacer importantes concesiones en relación con la apertura de su sector agropecuario y, después del retiro estadounidense, la eliminación de las estipulaciones problemáticas para los gobiernos de países en desarrollo facilitó la renegociación del texto del TPP para rebautizarlo como el Comprehensive and Progressive Trans Pacific Partnership Agreement (CPTPP).

\subsection{El RCEP}

El RCEP es la cristalización de los sucesivos proyectos de la EAC (propuesto por los gobiernos de la ASEAN como respuesta regional al NAFTA y a la UE), de la CEPEA respaldado por el Gobierno japonés como reacción a los problemas generados por la crisis asiática de 1997 y a la iniciativa ASEAN+3+3 (también impulsada por la ASEAN para establecer contra-equilibrios entre las potencias regionales).

Estos tres proyectos tenían como rasgo común la aspiración de los gobiernos de la ASEAN a formalizar de jure el proceso de integración económica regional. En el caso de la EAC, se trata de una iniciativa que buscaba abrir un espacio de negociación para el conjunto de gobiernos del sudeste asiático que, por separado, carecían de peso específico en la escena internacional. La CEPEA, por el contrario, era la propuesta de un Gobierno japonés que durante el último tercio del siglo xx había logrado desplazar a Estados Unidos como principal socio comercial de los países de Asia del este y del sureste. ASEAN+3+3 era, para los gobiernos de la Asociación, una forma de contrarrestar la influencia

3. Una lista detallada de las suspensiones es presentada por el Gobierno australiano en Department of Foreign Affairs and Trade, 2019. 
japonesa y china, mediante la inclusión de Corea, el tándem Australia-Nueva Zelanda e India en un esquema de integración regional progresiva.

El RCEP, también impulsado en el origen por la ASEAN, terminó convirtiéndose en el proyecto de integración regional del Gobierno chino; desde el inicio, el principal atractivo para los gobiernos participantes era la flexibilidad de la propuesta, pues permitía a cada gobierno adaptarse al proceso de apertura comercial de acuerdo con sus circunstancias particulares. ${ }^{4}$ El RCEP sanciona de jure el proceso de integración económica de facto que ha permitido a China desplazar a Japón como principal socio comercial de los países de la región; gracias a ese proceso, la economía china se ha convertido en el epicentro de un complejo productivo regional y este complejo, en gran medida, es un factor explicativo de la proyección internacional de la economía china.

Además, podemos considerar que para los gobiernos participantes el proyecto RCEP es un instrumento idóneo para contener a China, en la acepción del término de Richard M. Nixon (1967). En sentido negativo, la actitud del Gobierno indio confirma este juicio: mientras los gobiernos de ASEAN, de Japón, Corea, Australia y Nueva Zelanda buscaban crear un marco legal para establecer mecanismos de equilibrio con el Gobierno chino, el de India consideró mayores los perjuicios que los beneficios de sumarse al acuerdo.

\section{Contenido de los macroacuerdos comerciales}

Durante la negociación del TPP, un tema puesto de realce por los analistas internacionales fue el contenido del acuerdo: consideraban tan altos los estándares que una economía en desarrollo como la china no podría alcanzarlos durante un tiempo relativamente largo; por esa razón el TPP era considerado un instrumento político-comercial tendente al aislamiento de China (Kyu, 2015).

4. El modelo básico de la negociación fue el ASEAN-China Free Trade Agreement (ASEAN-Ch FTA), según el cual cada gobierno podía decidir: a) cuáles sectores productivos serían mantenidos al margen de la liberalización comercial por ser considerados sensibles; b) cuáles sectores serían sometidos a una liberalización rápida y cuáles a una relativamente lenta; c) los calendarios de la liberalización comercial serían diferenciados: más rápidos para ASEAN-6 (es decir, Brunei, Filipinas, Indonesia, Malasia, Singapur y Tailandia) y más lentos para ASEAN-4 (o sea, Camboya, Laos, Myanmar y Vietnam); d) la liberalización comercial iniciaría de manera inmediata con los productos agropecuarios, en el marco del Early Harvest Program, basado en las complementariedades existentes entre una economía china demandante de bienes agropecuarios y unas economías del sureste asiático productoras de ese tipo de productos (ASEAN, s. f.). 
En ese sentido, el TPP o su versión edulcorada del CPTPP ${ }^{5}$ podrían ser calificados como paradigmas de los macroacuerdos comerciales del Pacífico. En comparación con el contenido del CPTPP, el RCEP muestra importantes lagunas reveladoras de las debilidades estructurales no solo de la economía china, sino de la mayor parte de los participantes en la iniciativa; asimismo, pone en evidencia los límites comerciales infranqueables, por ahora, para la gran mayoría de los países en desarrollo participantes en el macroacuerdo asiático; en contraste, la comparación entre el CPTPP y el USMCA pone de realce, a la vez, nuevas reglamentaciones incluidas en el segundo acuerdo (tendentes a proteger los mercados estadounidense y norteamericano), así como vacíos temáticos que permiten entrever el desinterés de la administración Trump por temas que permitirían beneficiarse a los tres participantes en el USMCA.

\subsection{El contenido general}

Para fundar nuestro juicio anterior hemos construido la tabla 1, destacando los 16 temas de divergencia entre los tres acuerdos comerciales en cuestión. ${ }^{6}$ La primera evidencia es el enorme desfase del RCEP respecto a los otros dos acuerdos: el primer aspecto destacado es en relación con el CPTPP, pues el primero no incluye nueve temas presentes en el segundo y todos ellos son indicativos de la falta de madurez de la economía china para entrar en un régimen de libre comercio en el marco del proteccionismo consensuado, regulado por el texto del GATT de 1947; en términos precisos, de esos nueve temas, siete sí son cubiertos por el USMCA. ${ }^{7}$

5. Esto, en la medida en que el contenido general de los capítulos del TPP-12 fue retomado tal cual para la renegociación del CPTPP, eliminando únicamente los contenidos considerados incómodos por los gobiernos de países en desarrollo.

6. No hemos incluido el capítulo C.8 "Reconocimiento de la propiedad mexicana de los hidrocarburos", por ser de relevancia en la relación bilateral México-Estados Unidos, pero carente de importancia en el contexto amplio de la región del Pacífico.

7. Los siete temas no cubiertos por el RCEP, pero incluidos tanto en el CPTPP como en el USMCA, son: mercancías textiles y del vestido; defensa comercial; empresas propiedad del Estado y monopolios designados; trabajo; medio ambiente; competitividad y facilitación de negocios; transparencia y anticorrupción. Desarrollo y coherencia regulatoria son dos temas incluidos en el CPTPP, pero excluidos, a la vez, del RCEP y del USMCA. 


\section{Tabla 1}

Divergencias de contenido entre los macroacuerdos comerciales de la región del Pacífico

\begin{tabular}{|c|c|c|c|c|}
\hline \multirow{3}{*}{$\begin{array}{l}\text { Contenido temático de los capítulos seleccionados } \\
\text { Agricultura }\end{array}$} & \multicolumn{4}{|c|}{$\begin{array}{l}\text { Número del capítulo } \\
\text { en el índice del acuerdo }\end{array}$} \\
\hline & \multirow[t]{2}{*}{ RCEP } & \multirow[t]{2}{*}{ CPTPP } & \multicolumn{2}{|c|}{ USMCA } \\
\hline & & & C3 & \\
\hline Procedimientos de origen & & & C5 & \\
\hline Mercancías textiles y del vestido & & C4 & C6 & \\
\hline Defensa comercial & & C6 & $\mathrm{C} 10$ & \\
\hline Anexos sectoriales & & & C12 & * \\
\hline Empresas de propiedad del Estado y monopolios designados & & C17 & $\mathrm{C} 22$ & \\
\hline Laboral & & C19 & $\mathrm{C} 23$ & * \\
\hline Medio ambiente & & $\mathrm{C} 20$ & $\mathrm{C} 24$ & * \\
\hline Cooperación y desarrollo de capacidades & $\mathrm{C} 15$ & $\mathrm{C} 21$ & & \\
\hline Competitividad y facilitación de negocios & & $\mathrm{C} 22$ & $\mathrm{C} 26$ & * \\
\hline Desarrollo & & $\mathrm{C} 23$ & & \\
\hline Coherencia regulatoria & & $\mathrm{C} 25$ & & \\
\hline Transparencia y anticorrupción & & $\mathrm{C} 26$ & $\mathrm{C} 27$ & $*$ \\
\hline Buenas prácticas regulatorias & & & $\mathrm{C} 28$ & * \\
\hline Publicación y administración & & & C29 & \\
\hline Asuntos de política macroeconómica y tipos de cambio & & & C33 & $*$ \\
\hline
\end{tabular}

* Nuevo capítulo en relación con el contenido del North American Free Trade Agreement (NAFTA).

Fuente: elaboración propia con información de: New Zealand Foreign Affairs \& Trade Ministry (s. f.); Ministry of Foreign Affairs of Japan (2021); Office of de the us Trade Representative (s.f.).

El USMCA, por otra parte, incluye seis temas ${ }^{8}$ que a todas luces expresan intereses primordiales del Gobierno estadounidense y que fue más fácil hacerlos aceptar mediante acuerdos bilaterales que en negociaciones multilaterales. Todavía más, el USMCA también presenta lagunas en relación con los otros dos acuerdos comerciales: el caso más evidente es el tema de cooperación y desarrollo de capacidades, incluido tanto en el RCEP como en el CPTPP; éste es

8. Los seis temas citados son: agricultura, procedimientos de origen, anexos sectoriales, buenas prácticas regulatorias, publicación y administración, así como asuntos de política macroeconómica y tipos de cambio. 
uno de los temas favoritos de los gobiernos asiáticos y ello explica su inclusión en dos acuerdos con una fuerte participación asiática.

Por otra parte, como en el RCEP, en el USMCA tampoco fueron incluidos los temas desarrollo y coherencia regulatoria; una explicación racional es que estos tópicos corresponden a las relaciones horizontales de igualdad entre pares, propias de los acuerdos de libre comercio basados en el proteccionismo consensuado, como es el caso del CPTPP; ellos son, sin embargo, incompatibles con las relaciones verticales entre las autoridades de una economía considerada por ellas mismas superior a las economías de sus contrapartes (caso de Estados Unidos en el USMCA) o entre las autoridades de una economía protegida y sus socios comerciales (caso de China en el RCEP).

\subsection{La liberalización comercial en el CPTPP, el RCEP y el JUSTA}

Los representantes de la administración Trump han sido cuidadosos al eliminar la referencia al libre comercio de los acuerdos bilaterales establecidos con sus socios asiáticos y norteamericanos; en contraste, los gobiernos chino y japonés, así como los firmantes del CPTPP y del RCEP siguen reivindicando el libre comercio como el espíritu que los mueve; dicho en otras palabras: el proteccionismo consensuado sigue siendo el cimiento de ambos acuerdos.

Las dinámicas y los alcances de la liberalización comercial son, no obstante, muy diferentes, en función no solo del acuerdo comercial al cual se haga referencia, sino de los compromisos alcanzados entre dos o más signatarios de un acuerdo comercial específico; así, por ejemplo, en un acuerdo como el NAFTA los gobiernos signatarios se planteaban la creación de un área de libre comercio en un plazo de 10 años; con los acuerdos macrorregionales los plazos de liberalización comercial han sido extendidos, por regla general, a 20 años en el CPTPP y en el JUSTA. En el RCEP, no obstante, pesaron grandemente los antecedentes de los acuerdos bilaterales de la ASEAN con China; así, por ejemplo, la flexibilidad otorgada a los gobiernos para determinar cada uno la modalidad de la apertura comercial ha permitido que, por ejemplo, el Gobierno coreano acepte un plazo de 35 años para eliminar o reducir aranceles en algunos ítems específicos definidos por el Gobierno chino.

Por otra parte, ese tipo de flexibilidades ha permitido el surgimiento de nuevas barreras comerciales, aplicadas de manera diferenciada entre los signatarios de un acuerdo específico. Así, en el RCEP cada gobierno tiene la facultad de definir sectores productivos que fueron excluidos de la elimina- 
ción o reducción de tarifas; además, tiene la libertad de aplicar esa exclusión con algunas contrapartes específicas y de asignarles tarifas permanentes, tratándose de otras contrapartes. Además, en el JUSTA el Gobierno japonés impuso salvaguardas comerciales aplicables cuando sus importaciones provenientes sobrepasan determinados límites, mantuvo diferentes modalidades de cuotas de acceso al mercado japonés y recuperó las condiciones que rigen el trato de "nación más favorecida" (NMF) de acuerdo con las regulaciones del GATT de 1947.

Esto hace que, más allá de las profesiones de fe en materia de libre cambio por parte de los gobiernos participantes en el CPTPP o en el RCEP, el libre comercio sea entorpecido en áreas definidas por los intereses de los gobiernos signatarios y que para cada gobierno el alcance del libre cambio sea muy diferente, dependiendo del acuerdo y de cada contraparte. Japón es el arquetipo de esta filosofía pro libre mercado que se expresa de manera práctica en políticas comerciales diferenciadas, como podemos apreciar en la tabla 2. En efecto, en los renglones 1 y 2 de dicha tabla resumimos los alcances generales de la liberalización comercial otorgada por el Gobierno de Japón en el CPTPP, el RCEP y el JUSTA. El primer aspecto que debemos tomar en cuenta es el número de ítems sometido a las reglas de negociación de cada acuerdo:

El mayor número de ítems negociados fue registrado en el СРТPP: 9,406 ítems. Esto se debió a la presión estadounidense para negociar todos los sectores económicos. Ahora bien, de ésos, 7,686 (81.71\%) ítems serían liberalizados en el momento de la entrada en operación del acuerdo y 1,047 (11.13\%) ítems serían sometidos a una eliminación progresiva de aranceles en un plazo máximo de 20 años. Al término de este lapso, $92.84 \%$ de los ítems estarán libres de aranceles. Los 673 ítems restantes serían regulados mediante tarifas permanentes (135 ó $1.44 \%$ ), cuotas (236 ó $2.51 \%$ ) o el trato de NMF (302 ó 3.21\%).

El segundo número más grande de ítems negociados corresponde al RCEP: 8,715 ítems. En sí misma, esta cifra implica restricciones impuestas por el Gobierno japonés a las negociaciones con sus contrapartes en el acuerdo. La diferenciación de la política comercial nipona respecto a sus contrapartes, podemos apreciarla en dos sentidos: en primer lugar, a través de las concesiones comerciales otorgadas tanto a los gobiernos de la ASEAN como al de China: en uno y otro casos, 7,587 (87.06\%) y 7,360 (84.55\%) ítems serán libres de aranceles desde el inicio de operaciones del acuerdo o en un plazo máximo de 20 años. La segunda expresión de la política comercial japonesa 
trasluce en las formas de protección de los ítems no sujetos a la liberalización comercial; en el caso de la ASEAN, 732 (8.40\%) ítems fueron excluidos de las negociaciones y 396 (4.54\%) quedaron sujetos a aranceles permanentes; en contraste, para China los 1,355 (15.55\%) ítems restantes fueron excluidos de manera permanente de la reducción arancelaria.

En el caso del JUSTA, el número de ítems negociados por Japón fue mínimo: 621 ítems. Dos razones prácticas explican esta peculiaridad. Las negociaciones bilaterales tuvieron como punto de partida los resultados alcanzados en la negociación del TPP y la renegociación del CPTPP; en consecuencia, los dos gobiernos se concentraron sobre los temas más sensibles del comercio bilateral. La segunda razón práctica es la decisión bilateral para dejar fuera de la negociación el sector automotriz; en este sector específico, de acuerdo con el CPTPP podemos considerar que el Gobierno nipón ya había eliminado todas las barreras arancelarias y que, en contraste, el Gobierno estadounidense estaba interesado en buscar ventajas para acceder al mercado japonés de los vehículos automotores.

En todo caso, de los 621 ítems sometidos a negociación por el Gobierno nipón, 239 (38.49\%) quedarían libres de aranceles desde la entrada en vigor del acuerdo bilateral y 234 (37.68\%) serían sujetos a una reducción progresiva de los aranceles hasta suprimirlos en el plazo máximo de 20 años. Visto desde el otro ángulo: únicamente 138 ítems quedarían sujetos a diversos mecanismos de protección: 64 (10.31\%) tendrían una reducción progresiva de aranceles, pero protegidos por salvaguardas; siete (1.13\%) mantendrían tarifas permanentes; 25 (4.03\%) también quedarían sujetos a tarifas permanentes, reforzadas por salvaguardas en caso de una importación considerada excesiva por el Gobierno japonés; 34 (5.48\%) serían regulados por cuotas de acceso al mercado nipón; los 18 restantes serían sometidos al trato de NMF.

Un aspecto clave es que la negociación del JUSTA es la consecuencia necesaria de la negociación del TPP; por lo tanto, resulta válido considerar que de los 9,406 ítems sometidos a negociación por el Gobierno japonés, solo 138 (1.46\%) permanecerían sujetos a mecanismos proteccionistas en el marco del JUSTA; eso significaría que, en un plazo máximo de 20 años, el 98.54\% de los ítems incluidos por el Gobierno japonés en el CPTPP sería sometido a la liberalización comercial.

En resumen: la economía japonesa quedaría vinculada a la estadounidense mediante un régimen de libre cambio regulado mediante el JUSTA; las contrapartes de Japón en el CPTPP quedan sujetas a un reglamento comercial un poco 
más restrictivo; en cambio, los socios del RCEP estarán sujetos a restricciones todavía mayores para ASEAN y más agudas para China. La política comercial japonesa confirma nuestra tesis sobre el proteccionismo consensuado, considerado como la base de los llamados "acuerdos de libre comercio".

Hasta ahora concentramos nuestra atención sobre la estructura horizontal del total de ítems, de acuerdo con el mecanismo comercial utilizado por el Gobierno japonés. Pero, si fijamos nuestro interés en la estructura vertical de la tabla 2, es decir en los tipos de bienes o de los ítems, encontraremos que la política comercial japonesa corresponde a la de un país industrializado, carente de materias primas, pero cuyo Gobierno busca proteger hasta el último resquicio de su sector agropecuario: 
Tabla 2

Japón: ítems comerciales sujetos a los criterios de negociación en el CPTPP, el RCEP y el JUSTA

\begin{tabular}{|c|c|c|c|c|c|c|c|c|c|c|c|c|c|c|c|c|c|c|c|c|c|}
\hline & \multicolumn{6}{|c|}{ СРТPP } & \multicolumn{7}{|c|}{ RCEP } & \multicolumn{8}{|c|}{ JUSTA } \\
\hline & \multirow[b]{2}{*}{ Ítems } & \multirow[b]{2}{*}{$F$} & \multirow[b]{2}{*}{$r p$} & \multirow[b]{2}{*}{$T$} & \multirow[b]{2}{*}{$Q$} & \multirow[b]{2}{*}{$n m f$} & \multirow[b]{2}{*}{ Ítems } & \multicolumn{3}{|c|}{ asean } & \multicolumn{3}{|c|}{ China } & \multirow[b]{2}{*}{ Ítems } & \multirow[b]{2}{*}{$F$} & \multirow[b]{2}{*}{$r p$} & \multirow{2}{*}{\begin{tabular}{c|}
$R P+$ \\
$S G$
\end{tabular}} & \multirow[b]{2}{*}{$T$} & \multirow{2}{*}{\begin{tabular}{|c|}
$T+$ \\
$s g$
\end{tabular}} & \multirow[b]{2}{*}{$Q$} & \multirow[b]{2}{*}{$n m f$} \\
\hline & & & & & & & & $F$ & $U$ & $T$ & $F$ & $U$ & $T$ & & & & & & & & \\
\hline Total & 9,406 & 7,686 & 1,047 & 135 & 236 & 302 & 8,715 & 7,587 & 732 & 396 & 7,360 & 1,355 & & 621 & 239 & 234 & 64 & 7 & 25 & 34 & 18 \\
\hline $\begin{array}{l}\text { Mecanismos } \\
\text { comerciales } \\
\text { como \% del } \\
\text { total }\end{array}$ & 100 & 81.71 & 11.13 & 1.44 & 2.51 & 3.21 & 100 & 87.06 & 8.40 & 4.54 & 84.45 & 15.55 & & 100 & 38.49 & 37.68 & 10.31 & 1.13 & 4.03 & 5.48 & 2.90 \\
\hline Agropecuarios & 2,513 & 1,141 & 758 & 135 & 236 & 243 & 2,294 & 1,322 & 683 & 289 & 1,284 & 1,010 & & 593 & 215 & 230 & 64 & 7 & 25 & 34 & 18 \\
\hline $\begin{array}{l}\text { Sectores } 01 \text { a } \\
24, \% \text { del total }\end{array}$ & 26.72 & 14.85 & 72.40 & 100 & 100 & 80.46 & 26.32 & 17.42 & 93.31 & 72.98 & 17.45 & 74.54 & & 95.49 & 89.96 & 98.29 & 100 & 100 & 100 & 100 & 100 \\
\hline $\begin{array}{l}\text { Materias } \\
\text { primas de } \\
\text { origen mineral }\end{array}$ & 1,620 & 1,604 & 16 & & & & 1,423 & 1,416 & 5 & 2 & 1,374 & 49 & & 28 & 24 & 4 & & & & & \\
\hline $\begin{array}{l}\text { Sectores } 25 \text { a } \\
40, \% \text { del total }\end{array}$ & 17.22 & 20.87 & 1.53 & & & & 16.33 & 18.66 & 0.68 & 0.51 & 18.67 & 3.62 & & 4.51 & 10.04 & 1.71 & & & & & \\
\hline $\begin{array}{l}\text { Materias } \\
\text { primas } \\
\text { de origen } \\
\text { agropecuario }\end{array}$ & 616 & 369 & 216 & & & 31 & 652 & 546 & 32 & 74 & 452 & 200 & & & & & & & & & \\
\hline $\begin{array}{l}\text { Sectores } 41 \text { a } \\
49, \% \text { del total }\end{array}$ & 6.55 & 4.80 & 20.63 & & & 10.26 & 7.48 & 7.20 & 4.37 & 18.69 & 6.14 & 14.76 & & & & & & & & & \\
\hline $\begin{array}{l}\text { Textil, vestido y } \\
\text { calzado }\end{array}$ & 1,997 & 1,947 & 22 & & & 28 & 1,796 & 1,753 & 12 & 31 & 1,725 & 71 & & & & & & & & & \\
\hline $\begin{array}{l}\text { Sectores } 50 \text { a } \\
67, \% \text { del total }\end{array}$ & 21.23 & 25.33 & 2.10 & & & 9.27 & 20.61 & 23.11 & 1.64 & 7.83 & 23.44 & 5.24 & & & & & & & & & \\
\hline
\end{tabular}




\begin{tabular}{|c|c|c|c|c|c|c|c|c|c|c|c|c|c|c|c|c|c|c|c|c|}
\hline & \multicolumn{6}{|c|}{ CPTPP } & \multicolumn{6}{|c|}{ RCEP } & \multicolumn{8}{|c|}{ JUSTA } \\
\hline & \multirow[b]{2}{*}{ Ítems } & \multirow[b]{2}{*}{ F } & \multirow[b]{2}{*}{$r p$} & \multirow[b]{2}{*}{$T$} & \multirow[b]{2}{*}{$Q$} & \multirow[b]{2}{*}{$n m f$} & \multirow[b]{2}{*}{ Ítems } & \multicolumn{3}{|c|}{ asean } & \multicolumn{2}{|c|}{ China } & \multirow[b]{2}{*}{ Ítems } & \multirow[b]{2}{*}{$F$} & \multirow[b]{2}{*}{$r p$} & \multirow{2}{*}{$\frac{R P+}{S G}$} & \multirow[b]{2}{*}{$T$} & \multirow{2}{*}{\begin{tabular}{l|l|l}
$T+$ \\
$s g$
\end{tabular}} & \multirow[b]{2}{*}{$Q$} & \multirow[b]{2}{*}{$n m f$} \\
\hline & & & & & & & & $F$ & $U$ & $T$ & $F$ & $U$ & & & & & & & & \\
\hline $\begin{array}{l}\text { Piedra, vidrio y } \\
\text { metales }\end{array}$ & 1,103 & 1,074 & 29 & & & & 1,280 & 1,280 & & & 1,258 & 22 & & & & & & & & \\
\hline $\begin{array}{l}\text { Sectores } 68 \text { a } \\
83, \% \text { del total }\end{array}$ & 11.73 & 13.97 & 2.77 & & & & 14.69 & 16.87 & & & 17.09 & 1.62 & & & & & & & & \\
\hline Manufacturas & 1,557 & 1,551 & 6 & & & & 1,270 & 1,270 & & & 1,267 & 3 & & & & & & & & \\
\hline $\begin{array}{l}\text { Sectores } 84 \text { a } \\
97, \% \text { del total }\end{array}$ & 16.55 & 20.18 & 0.57 & & & & 14.57 & 16.74 & & & 17.21 & 0.22 & & & & & & & & \\
\hline \multicolumn{21}{|c|}{$\begin{array}{l}\mathrm{F}=\text { libre de aranceles desde la entrada en vigor del acuerdo; en los casos de China y Japón, en el marco del RCEP también incluye los ítems RP, cuyos aranceles son } \\
\text { eliminados progresivamente, en diferentes plazos que tienen como máximo } 20 \text { años. }\end{array}$} \\
\hline \multicolumn{21}{|c|}{ RP = eliminación de aranceles en plazos que tienen como máximo 20 años (la excepción son los 35 años que deberá esperar Corea para acceder al mercado chino). } \\
\hline \multicolumn{21}{|c|}{ SG = salvaguardas, aplicables cuando las importaciones japonesas provenientes de Estados Unidos sobrepasan cuotas previamente especificadas. } \\
\hline \multicolumn{21}{|c|}{$\mathrm{T}$ = tarifas prevalecientes de manera permanente después de transcurridos diferentes plazos durante los cuales los aranceles son progresivamente reducidos. } \\
\hline \multicolumn{21}{|c|}{$\mathrm{Q}=$ cuotas de acceso al mercado japonés. } \\
\hline \multicolumn{21}{|c|}{ NMF = tratamiento basado en el principio de la nación más favorecida, de acuerdo con las regulaciones del GATT. } \\
\hline \multicolumn{21}{|c|}{ U = ítems excluidos de la eliminación y de la reducción de aranceles. } \\
\hline \multicolumn{21}{|c|}{$\begin{array}{l}\text { Fuente: elaboración propia, con informaciones provenientes de los calendarios para la reducción o eliminación de tarifas, propuesto por } \\
\text { cada gobierno en el marco de cada uno de los acuerdos comerciales regionales. }\end{array}$} \\
\hline
\end{tabular}


El carácter de Japón como una economía industrializada destaca en el JUSTA y en el CPTPP; la tabla 2 permite ver que en ambos casos, después del plazo para la reducción arancelaria progresiva, solo quedarán sujetos a mecanismos de protección los ítems correspondientes al sector agropecuario. En contraste, en el marco del RCEP el Gobierno japonés mantiene mecanismos proteccionistas en áreas de las materias primas de origen mineral y agropecuario, así como en las de las industrias textil, vestido y calzado en el caso de la ASEAN; en el de China, las exclusiones para la negociación se extienden a todo el espectro de bienes de origen chino.

La carencia en Japón de materias primas de origen mineral es una característica que suele ser evocada como un factor explicativo de la internacionalización de la economía japonesa desde los inicios de la industrialización, durante la restauración Meiji. Ahora, en calidad de economía industrializada, abierta al libre comercio con sus principales socios comerciales del Pacífico, el Gobierno japonés vuelve a poner de realce esa característica de la economía nipona mediante la liberalización de las importaciones de ese tipo particular de bienes. Por supuesto, la apertura será total en el marco del JUSTA, con algunas restricciones puntuales en el CPTPP y con mayores reservas en el RCEP (más marcadas para el Gobierno chino que para los de la ASEAN).

Finalmente, sin importar la filiación política de quien lo encabece, para el Gobierno japonés la protección del sector agropecuario es, en términos políticos, una cuestión crucial; debido a ello es evidente que en los tres acuerdos comerciales considerados, el mayor número, absoluto y relativo, de mecanismos proteccionistas prevalece para los bienes provenientes del sector agropecuario. Sin embargo, como hemos visto, en el caso del JUSTA esos mecanismos proteccionistas sectoriales han sido reducidos a su mínima expresión.

\subsection{Las ventajas estratégicas de cada proyecto macrorregional}

Finalmente, líneas arriba mencionamos que los asesores comerciales de Donald Trump decidieron cambiar las reglas del juego de la competencia en los mercados internacionales; en efecto, por regla general la noción de ventaja comparativa definida por David Ricardo (2014) indica que un país debe especializarse en las actividades donde tiene más ventajas relativas (a diferencia de Adam Smith, quien privilegia las ventajas absolutas). Las teorías del desarrollo económico recuperaron esa noción ricardiana, para proponer la producción industrial como el medio para superar el atraso económico; 
conforme progresaba el desarrollo tecnológico, las economías industrializadas fueron especializándose en la producción y exportación de bienes con contenidos cada vez mayores de valor agregado y de tecnología; la formación de capital es, en consecuencia, la condición sine qua non de la industrialización.

Si miramos con atención la expresión de ese indicador (tabla 3) en cada uno de los tres megaproyectos de integración económica, veremos que el RCEP tiene una gran ventaja sobre los otros dos: de acuerdo con las cifras de 2018 , concentraba el $41.16 \%$ de la formación bruta de capital total en la escala mundial, seguido por el proyecto transregional de la administración Trump (29.93\% del total mundial) y, muy detrás, por el CPTPP (11.62\% del total mundial). Ahora bien, el desempeño comercial internacional está en relación directa con la formación bruta de capital; así, es normal que el RCEP aporte el $29.37 \%$ del comercio total mundial y que sea seguido por el proyecto de la administración Trump (22.52\%) y por el CPTPP (14.48\%). Hasta aquí, todo coincide con la teoría ricardiana de la ventaja comparativa: el éxito en la producción de bienes comerciales depende de la especialización en su producción y, en última instancia, de la inversión productiva.

Ahora bien, la cuestión es que la "formación bruta de capital" es uno de los rubros que integran el PIB, desde el punto de vista del consumo. Los otros dos componentes del consumo son el "consumo de los hogares" y el "consumo del gobierno"; si introducimos estas dos variables en la ecuación, comprenderemos que el consumo productivo como "formación bruta de capital" destinada a la producción de bienes exportables solo tiene razón de ser si la demanda externa es adecuada para absorber esos bienes. Es decir, la formación bruta de capital depende del consumo de los hogares y de los gobiernos en otros países, y los asesores comerciales de Trump decidieron utilizar el consumo estadounidense como una ventaja competitiva para negociar los acuerdos bilaterales con sus contrapartes; las estadísticas muestran que, en 2018, el proyecto de la administración Trump concentraba 39.42 y 33.05\% de los totales mundiales del consumo de los hogares y del consumo del Gobierno; le seguían el RCEP, con proporciones del 24.55 y $29.09 \%$, y el CPTPP con participaciones del 12.93 y $14.03 \%$ (véase tabla 3 ).

En la escala de los países y teniendo en cuenta los promotores de cada uno de los tres proyectos de integración económica, debemos tener en cuenta que Estados Unidos concentraba el 28.66 y $20.60 \%$ de los totales mundiales del consumo tanto de los hogares como de los gobiernos; las proporciones correspondientes a China, el gigante exportador asiático, eran 11.57 y 15.46 , 
y las de Japón eran 5.41 y $6.74 \%$ (United Nations, s. f.). Sobre esta base factual es comprensible que los estrategas comerciales estadounidenses hayan decidido cambiar las reglas con que juegan en el mercado mundial: quienes deseen vender sus productos en los mayores mercados de consumo efectivo, estadounidense o norteamericano, deben sujetarse a las reglas impuestas en los acuerdos bilaterales o en las reglas de origen del USMCA; las regulaciones para acceder a uno y otro ámbitos están diseñadas para reducir los déficits comerciales bilaterales de la economía estadounidense.

\section{Tabla 3}

Participación de los integrantes de los tres proyectos transregionales del Pacífico en los componentes del consumo del PIB, 2018

\begin{tabular}{l|l|l|l|l|l}
\hline & $\begin{array}{l}\text { Consumo } \\
\text { hogares }\end{array}$ & $\begin{array}{l}\text { Consumo } \\
\text { gobierno }\end{array}$ & $\begin{array}{l}\text { Formación } \\
\text { bruta de capital }\end{array}$ & $\begin{array}{l}\text { Comercio } \\
\text { total }\end{array}$ & PIB \\
\hline Total mundial & $46,535,129$ & $13,216,225$ & $21,344,885$ & $46,914,832$ & $81,222,806$ \\
\hline CPTPP & $6,016,111$ & $1,854,625$ & $2,480,679$ & $6,792,095$ & $10,384,403$ \\
\hline \% del total & 12.93 & 14.03 & 11.62 & 14.48 & 12.79 \\
\hline Proyecto EUA & $18,438,431$ & $4,367,348$ & $6,388,011$ & $10,565,066$ & $28,557,226$ \\
\hline \% del total & 39.62 & 33.05 & 29.93 & 22.52 & 35.16 \\
\hline RCEP & $11,425,391$ & $3,845,044$ & $8,785,518$ & $13,779,061$ & $24,289,849$ \\
\hline \% del total & 24.55 & 29.09 & 41.16 & 29.37 & 29.91 \\
\hline Cifras en millones de dólares, en precios de 2015.
\end{tabular}

Fuente: elaboración propia con información de United Nations (s. f.). https://unstats.un.org/ unsd/snaama

Por supuesto, la apuesta por el consumo para reactivar las economías de América del Norte puede ser efectiva en el corto y mediano plazos; en el largo, la formación de capital terminará por imponer las ventajas del incremento de la productividad del trabajo; en ese sentido, los gobiernos de América del Norte pueden usar el consumo como palanca para atraer inversiones productivas; pero, el peor error sería entender que el consumo puede sustituir de manera permanente la inversión productiva.

\section{Conclusiones}

Viendo con detalle algunas de las características de los componentes del proyecto transregional de integración de la administración Trump, del CPTPP y 
del RCEP, podemos decir que aquél mantiene ventajas sobre éstos y que esas ventajas benefician exclusivamente la economía estadounidense; para las contrapartes del Gobierno estadounidense los beneficios de la relación con Estados Unidos son parciales y, en gran medida, potenciales. Para comprender cómo esas potencialidades pueden devenir realidades, necesitamos tener en consideración que uno de los principales objetivos de la administración Trump era presionar el proceso de globalización hacia una regionalización que permitiese la afluencia de inversiones directas de las economías avanzadas de Asia del Pacífico (pero también de Europa) hacia las economías de Estados Unidos y de América del Norte.

En ese marco, el Gobierno estadounidense cuenta ahora con los medios (los acuerdos con sus contrapartes asiáticas y norteamericanas) para inducir la transición de la globalización a la regionalización. Para consolidar ese proceso todavía sería necesario cerrar el círculo de los acuerdos comerciales bilaterales; es decir, completarlos mediante la renegociación del Acuerdo de Asociación Económica México-Japón y la negociación de un acuerdo bilateral MéxicoCorea, por un lado, y, por otro, la renegociación del Acuerdo de Libre Comercio Canadá-Corea y la negociación de un acuerdo bilateral Canadá-Japón.

Cierto, los Gobiernos mexicano y canadiense son socios del japonés en el СРTPP; sin embargo, en la perspectiva estadounidense el CPTPP es un acuerdo en competencia con su propia iniciativa; además, su lógica comercial (así como la de los acuerdos bilaterales México-Japón y Canadá-Corea) es radicalmente diferente de la de los acuerdos bilaterales negociados por la administración Trump. En ese sentido, los Gobiernos mexicano y canadiense necesitan redefinir los mecanismos que regulan sus vínculos con sus contrapartes asiáticas, para adecuarlos a las nuevas circunstancias.

Para japoneses y coreanos la capacidad de consumo del mercado estadounidense (pero también del mercado norteamericano) es clave en la medida en que, en sus exportaciones directas, predominan los bienes de consumo con alto contenido tecnológico y de valor agregado; los mercados asiáticos, en general, todavía carecen de la madurez suficiente para transitar al consumo masivo de ese tipo de bienes, y en el mercado chino las empresas locales tienden a sustituir la foráneas para satisfacer la demanda creciente de bienes de esa naturaleza; en consecuencia, la afinidad entre las bases productivas japonesa y coreana es mayor con la capacidad de consumo de Estados Unidos (y América del Norte). 
Queda por ver si los responsables de las decisiones en materia de política económica internacional de las contrapartes de Estados Unidos, en Asia del Pacífico y América del Norte están a la altura de las circunstancias. Además, debemos tener en cuenta dos factores extraeconómicos que habrán de influir en la reorganización del sistema económico mundial: el primer factor es la pandemia del SARS-CoV-2 y las presiones que ejercerá sobre las empresas transnacionales para abandonar el esquema de las cadenas globales de valor, para adoptar otro orientado hacia la regionalización tanto de la producción como de la comercialización; esas presiones serán más grandes, mientras la pandemia se extienda en el tiempo y permanezca fuera de control, obligando a cierres recurrentes de las actividades productivas en regiones geográficas amplias.

El segundo factor es la política económica internacional de la administración Biden. La antipatía resentida, urbi et orbi, por Donald Trump ha influido en el sentimiento de que el cambio de administración en Estados Unidos implicará un retorno a la situación pre-Trump. Eso quizá sea posible en algunos campos de la política exterior estadounidense, pero en el de la política comercial, nuestra apreciación es que Biden no solo habrá de apoyarse en los acuerdos negociados por su predecesor, sino que podría poner en práctica una política comercial internacional todavía más restrictiva; en ese sentido, sería de esperar la culminación de las negociaciones con Japón y la reconducción de las iniciadas con las instancias políticas europeas.

En todo caso, para las clases empresarial y política mexicanas, acostumbradas al proteccionismo económico, el USMCA, en particular, y el proyecto estadounidense de integración transregional, en sentido más amplio, representan una oportunidad única para reactivar una economía fuertemente afectada por la recesión global, siempre y cuando sean capaces de adaptarse a las nuevas circunstancias creadas por la redefinición de las relaciones del Gobierno estadounidense con sus contrapartes estratégicas.

\section{Referencias bibliográficas}

Apec. (2008). 2008 Leaders' Declaration. https://www.APEC.org/MeetingPapers/Leaders-Declarations/2008/2008_aelm

AsEan. (2020). Joint Leader's Statement on The Regional Comprehensive Economic Partnership (RCEP). https://asean.org/joint-leaders-statementregional-comprehensive-economic-partnership-RCEP-2/ 
ASEAN. (s.f.). ASEAN-China Free Trade Area. Building Strong Economic Partnerships. https://www.asean.org/storage/images/2015/October/outreachdocument/Edited\%20ACFTA.pdf

Department of Foreign Affairs and Trade. (2019). СPTPP suspensions explained. Australian Government. https://www.dfat.gov.au/trade/agreements/inforce/CPTPP/outcomes-documents/Pages/CPTPP-suspensions-explained

Kyu, P. (2015, octubre 19). How China's exclusion form the TPP could hurt its economic growth. Fortune. https://fortune.com/2015/10/19/chinaexclusion-tpp-economic-growth/

Ministry of Foreign Affairs of Japan. (2021, febrero 8.). Text of Regional Comprehensive Economic Partnership (RCEP) Agreement. https://www.mofa. go.jp/policy/economy/page1e_kanri_000001_00007.html

New Zealand Foreign Affairs \& Trade Ministry. (s.f.). Comprehensive and Progressive Agreement for Trans-Pacific Partnership text and resources. https:// www.mfat.govt.nz/en/trade/free-trade-agreements/free-trade-agreements-in-force/comprehensive-and-progressive-agreement-for-transpacific-partnership-CPTPP/comprehensive-and-progressive-agreementfor-trans-pacific-partnership-text-and-resources/

Nixon, R. M. (1967). Asia after Viet Nam. Foreign Affairs, 46(1). https:// www.foreignaffairs.com/articles/united-states/1967-10-01/asia-afterviet-nam

Office of the us Trade Representative. (2019). U. S.-Japan Trade Agreement Text. Disponible https://ustr.gov/countries-regions/japan-korea-APEC/ japan/us-japan-trade-agreement-negotiations/us-japan-trade-agreement-text

Office of the US Trade Representative. (s.f.). Agreement between the United States of America, The United Mexican States, and Canada 7/1/20 Text. https://ustr.gov/trade-agreements/free-trade-agreements/united-statesmexico-canada-agreement/agreement-between

Palmer, D. (2013, febrero 20). US says Japan must negotiate on rice to join trade talks. Reuters. https://www.reuters.com/article/usa-japan-kirk/ update-2-u-s-says-japan-must-negotiate-on-rice-to-join-trade-talksidUSL1N0BK5U720130220

Ramírez Bonilla, J. J. (2020a). Bye bye Latin America? México y la política económica internacional de la administración Trump. En Observatorio América Latina-Asia Pacífico, América Latina y Asia: Entre la revolución digital y la globalización cuestionada (pp. 146-177). ALADI; CAF; CEPAL. 
https://www.observatorioasiapacifico.org/images/publicaciones/Cuarto_Seminario_Academico/Libro_IV_Seminario_Final.pdf

Ramírez Bonilla, J. J. (2020b). La administración Trump y la transición del proteccionismo consensuado al proteccionismo coercitivo. En E. TziliApango, J. L. León-Manríquez \& G. Pérez-Gavilán (Eds.). Asia Pacífico: Poder y prosperidad en la era de la desglobalización (pp. 237-294). http://dcsh. xoc.uam.mx/repdig/index.php/libros-dcsh/politica-y-cultura/ítem/337asia-pacifico-poder-y-prosperidad-en-la-era-de-la-desglobalizacion

Reuters. (2018, octubre 11). USTR to Congress: US wants to open trade talks with EU, UK, Japan, Philippines. https://www.reuters.com/article/us-usa-tradeustr/ustr-to-congress-u-s-wants-to-open-trade-talks-with-eu-uk-japanphilippines-idUSKCN1ML2G2

Ricardo, D. (2014). Principios de economía política y tributación. Fondo de Cultura Económica.

Ruiz, C. (2020, noviembre 18). RCEP vs TMEC: Un nuevo frente de debate. El Financiero. https://www.elfinanciero.com.mx/opinion/clemente-ruizduran1/RCEP-vs-tmec-un-nuevo-frente-de-debate

Trump, D. (2016). Transcript of Donald Trump's Foreign Policy Speech, April 27, 2016. The Fiscal Times. https://www.thefiscaltimes.com/2016/04/28/ Transcript-Donald-Trump-s-Foreign-Policy-Speech-April-27-2016

Trump, D. (2017). Remarks by President Trump at APEC CEO Sumiit, Da Nang, Vietnam. The White House. https://trumpwhitehouse.archives.gov/ briefings-statements/remarks-president-trump-APEC-ceo-summit-danang-vietnam/

United Nations. (2019). 2019 Revision of World Population Prospects. https:// population.un.org/wpp/

United Nations. (s.f.). National Accounts - Analysis of Main Aggregates (AMA). https://unstats.un.org/unsd/snaama 